\title{
Long-Term Management of RAP Lesions in Clinical Practice: Treatment Efficacy and Predictors of Functional Improvement
}

\author{
Marco Frederico Marques ${ }^{a}$ João Pedro Marques ${ }^{a, b}$ João Quadrado Gila, b \\ José Costa ${ }^{a}$ Elisabete Almeida ${ }^{a}$ Maria da Luz Cachulo ${ }^{a-c}$ Isabel Pires ${ }^{a-c}$ \\ João Figueira ${ }^{\mathrm{a}-\mathrm{c}}$ Rufino Silva ${ }^{\mathrm{a}-\mathrm{c}}$ \\ aDepartment of Ophthalmology, Centro Hospitalar e Universitário de Coimbra (CHUC), ${ }^{b}$ Association for Innovation \\ and Biomedical Research on Light and Image (AIBILI), and ' Faculty of Medicine, University of Coimbra (FMUC), \\ Coimbra, Portugal
}

\section{Key Words}

Age-related macular degeneration - Anti-angiogenic agents . Choroidal neovascularization - Neovascular phenotypes .

Photodynamic therapy · Ranibizumab · Retinal angiomatous proliferation - Retinal neovascularization .

Type 3 neovascularization

\begin{abstract}
Purpose: To evaluate the long-term efficacy of ranibizumab in the treatment of retinal angiomatous proliferation (RAP) and to identify predictors of functional outcome. Methods: Retrospective case series comprised 79 eyes of 68 consecutive patients with RAP followed up $\geq 36$ months. Primary end-points were best-corrected visual acuity (BCVA) and central macular thickness (CMT) variation at 36 months and at the last visit. Results: Mean follow-up time was $59.8 \pm 16.0$ months. All eyes were treated with pro re nata ranibizumab, with $(n=33)$ or without $(n=46)$ photodynamic therapy (PDT). Stabilization or improvement in BCVA was observed in $50.6 \%$ of the patients at 36 months, and in $40.5 \%$ at the end of the follow-up, where $20.3 \%$ preserved reading vision. A significant decrease in CMT was observed at 36 months $(p<0.001)$, but not at the end of the follow-up. Geographic atrophy (GA) was present in $59.5 \%$ of the eyes at the final
\end{abstract}

visit. Baseline subretinal fluid was associated with better visual outcomes $(p=0.001)$. Results of combination treatment with intravitreal ranibizumab and PDT did not significantly differ from ranibizumab monotherapy. Conclusion: Modest functional outcomes can be expected from the long-term treatment of RAP lesions in clinical practice, most likely due to the advent of GA. Baseline subretinal fluid positively correlated with final BCVA.

(c) 2015 S. Karger AG, Basel

\section{Introduction}

Retinal angiomatous proliferation (RAP), also known as type 3 neovascularization, is a well-recognized neovascular phenotype of age-related macular degeneration (AMD), accounting for $12-15 \%$ of its exudative form in Caucasians [1]. The angioproliferative process associated with RAP diverges from other neovascular membranes, as it is believed to start deeply within the retina [2-6]. Yannuzzi et al. [7] first classified RAP lesions into three stages according to clinical and angiographic findings: stage 1, intraretinal neovascularization; stage 2, intraretinal plus subretinal neovascularization, and stage 3 , choroidal neovascularization.

\section{KARGER}

E-Mail karger@karger.com

www.karger.com/ore
(C) 2015 S. Karger AG, Basel

0030-3747/15/0553-0119\$39.50/0
Marco Frederico Marques

Hospitais da Universidade de Coimbra

R. Fonseca Pinto

PT-3000-075 Coimbra (Portugal)

E-Mail mfolivmarques@gmail.com 
Fig. 1. Top left: baseline CFP of one of the RAP patients included in the study. Note the paucity of drusen, the presence of pigmentary changes in the macular area as well as an apparently thickened retina. Baseline time-domain OCT of this eye shows intraretinal fluid and the presence of a retinal pigment epithelium detachment (bottom left). On the right, central GA can be seen both in infrared imaging (top) and spectral-domain OCT (bottom).
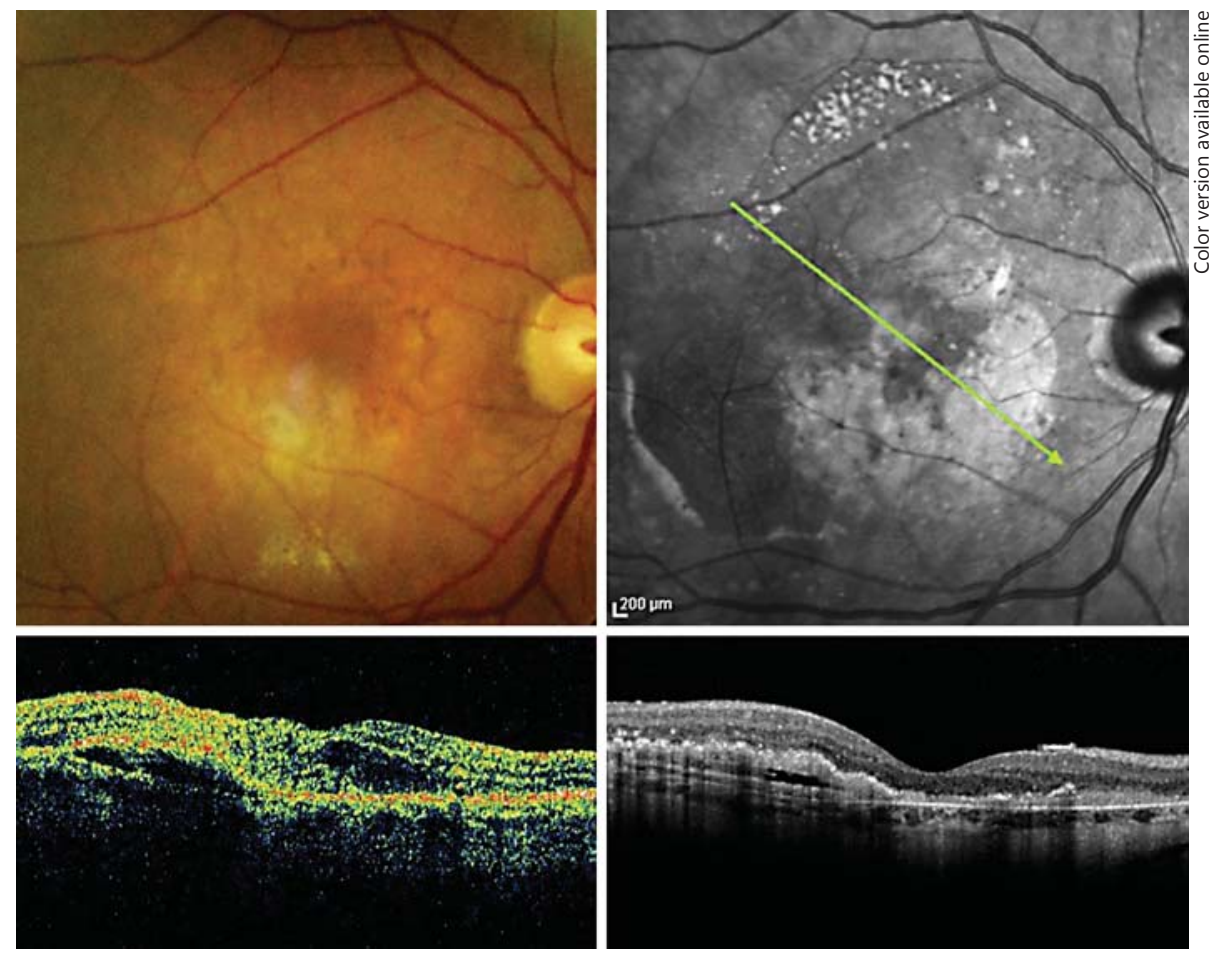

The advent of optical coherence tomography (OCT), fundus autofluorescence and infrared imaging allowed for a better characterization of the structural changes associated with the disease, such as drusen, reticular pseudodrusen, inner and outer retinal cysts, pigment epithelium detachment (either with or without fibrovascular material), subretinal fluid and geographic atrophy (GA) $[8,9]$. The prognostic value of some of these features has been highlighted in several publications $[8,10-12]$.

RAP has historically been coined a bilaterally aggressive disease with a poor natural history and high relapse rates. While several treatment approaches proved unsuccessful in the past [13-17], the blockage of vascular endothelium growth factor (VEGF) has substantially changed the prognosis of this condition. Reports on intravitreal (IV) ranibizumab, either in monotherapy or with concomitant photodynamic therapy (PDT), have underlined the responsiveness of RAP lesions to both treatment modalities [18-21]. However, standard protocols for the management of the disease are currently not available [1], and published data are limited to small case series with short follow-up periods [18-21].

The purpose of this study was to evaluate the longterm efficacy and safety of IV ranibizumab in eyes with RAP treated in a tertiary care hospital and to identify predictive factors of functional outcome.

\section{Materials and Methods}

\section{Population}

Retrospective case series included 79 eyes of 68 consecutive RAP patients followed from January 2007 to October 2011. Inclusion criteria were a confirmed diagnosis of RAP based on clinical and angiographic findings and $\geq 36$ months of follow-up after the first IV ranibizumab injection. Patients previously on other AMD treatment formulations, those with a positive past medical history for acute cardiovascular events and those with ophthalmologic conditions likely to jeopardize the clinical assessment were excluded from this analysis. Baseline best-corrected visual acuity (BCVA) was not set as an inclusion or exclusion criterion. Before inclusion, all patients signed an informed consent and the study was conducted according to the tenets of the Declaration of Helsinki.

\section{Clinical Assessment and Imaging}

Baseline assessment included a complete ophthalmologic examination complemented with color fundus photography (CFP; fig. 1), fluorescein angiography, indocyanine green angiography and OCT. BCVA was measured according to a standardized refraction protocol using an Early Treatment Diabetic Retinopathy study (ETDRS) chart. Visual enhancement and deterioration were defined as an increase or decrease $\geq 5$ ETDRS letters from baseline, respectively. A stabilized BCVA encompassed any oscillation between those values. Reading vision was classified as a BCVA $\geq 20 / 70$ (Snellen visual acuity chart) according to the World Health Organization criteria. For standardization purposes, we will refer to this reading vision as an ETDRS equivalent of $\geq 60$ letters. RAP stage at diagnosis was categorized by a senior retina specialist according to the three angiogenic stages defined by Yannuzzi et al. 


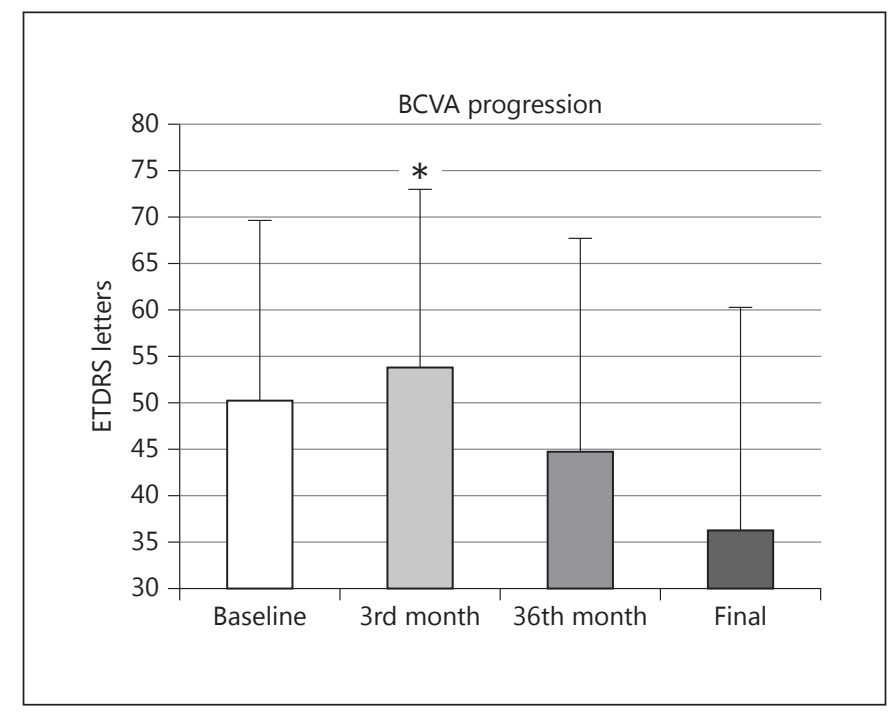

Fig. 2. BCVA progression during follow-up. ${ }^{*} \mathrm{p}<0.05$.

[7]. Before 2009, all OCT images were obtained using the highresolution 6-radial line protocol of Stratus ${ }^{\circledR}$ OCT (Carl Zeiss Meditec, Dublin, Calif., USA). With the availability of spectral-domain OCT, all further images were acquired using the Spectralis ${ }^{\circledR}$ OCT (Heidelberg Engineering, Heidelberg, Germany). Since both spectral- and time-domain OCT examinations were performed, timedomain OCT grading was calibrated to spectral-domain OCT to minimize potential measurement bias [22]. Identification of GA was based on the presence of $>1$ patch of $>250 \mu \mathrm{m}$ in the lengthiest linear dimension in CFP within the macula, evidencing $>1$ of these additional characteristics: sharply delimited boundaries, excavated or punched-out appearance on stereoscopy, enhancement of the choroidal circulation in CFP and correlation with OCT findings. BCVA and central macular thickness (CMT) were recorded during every visit. Adverse events possibly related with IV ranibizumab administration were registered.

\section{Treatment}

All patients were treated with IV ranibizumab (Lucentis ${ }^{\circledR}$; Novartis International AG, Basel, Switzerland), $0.5 \mathrm{mg} / 0.05 \mathrm{ml}$, in a nonloading dose pro re nata $(\mathrm{PRN})$ regimen, with $(\mathrm{n}=33)$ or without $(\mathrm{n}=46)$ associated PDT with verteporfin (Visudyne ${ }^{\circledR}$; Novartis International AG). Decision on retreatment was based on a decrease in BCVA $\geq 1$ ETDRS line and/or OCT findings, specifically the presence of retinal pigment epithelium detachment, hemorrhage, and intra- or subretinal fluid. Each IV injection was performed $3.5-4.0 \mathrm{~mm}$ posterior to the corneal limbus using a 30 -gauge needle after instillation of topical anesthesia and 5\% povidone-iodine solution in the fornix. Prophylaxis of endophthalmitis was achieved with topical quinolone q.i.d. 4 days before and 4 days after the IV injection. PDT was performed only in cases included until December 2009, and discontinued after an intermediate analysis showed no additional benefit versus PRN IV ranibizumab monotherapy. When treating bilateral RAP, IV ranibizum$\mathrm{ab}$ or PDT were performed on separate days.

Long-Term Management of RAP Lesions

\section{End-Points}

BCVA and CMT variation at 36 months and at the end of the follow-up were defined as primary end-points of IV ranibizumab efficacy.

\section{Statistical Analysis}

All statistical analyses were performed using STATA ${ }^{\circledR}$ version 12.1 (StataCorp LP, College Station, Tex., USA), and $\mathrm{p} \leq 0.05$ was considered statistically significant. Categorical variables were summarized as frequencies and percentages, and numerical variables as means \pm SD and minimum/maximum. The Shapiro-Wilk test was used to assess the normal distribution of continuous variables. The $\chi^{2}$ test or Fisher's exact test was performed to appraise associations between categorical variables, and the Student t test for associations involving independent and paired samples. MannWhitney, Wilcoxon, ANOVA and Kruskal-Wallis tests were applied for associations between continuous variables.

\section{Results}

\section{Demographics}

Seventy-nine eyes from 68 individuals were analyzed; 45 of the patients were females. Mean age at diagnosis was $78.9 \pm 7.0$ years (range: $60-92$ years). Follow-up lasted at least 3 years: $72.2 \%(n=57)$ had $\geq 4$ years and $48.1 \%(n=$ 38) $\geq 5$ years, with a mean follow-up of $59.8 \pm 16.0$ months.

\section{RAP Stage and Neovascularization of the Fellow Eye}

According to the classification proposed by Yannuzzi et al. [7], $13(16.5 \%)$ eyes were classified at baseline as stage $1,31(39.2 \%)$ as stage 2 and $35(44.3 \%)$ as stage 3. Stage 3 eyes presented a significantly lower baseline BCVA when compared to eyes in stage $1(p=0.022)$ or stage $2(\mathrm{p}=0.010)$. However, no substantial differences were noticed in the total number of IV ranibizumab injections $(\mathrm{p}=0.220)$ or presence of GA at the final visit $(\mathrm{p}=$ 0.206) among the different RAP stages. Thirty-two $(41.5 \%)$ patients had unaffected fellow eyes at baseline, and 17 (51.3\%) of them developed neovascularization during the follow-up.

\section{Visual Acuity}

BCVA progression during follow-up and visual outcome at 36 months and at the end of the follow-up are depicted in figures 2 and 3. At diagnosis, 20.3\% $(\mathrm{n}=16)$ of the patients were legally blind ( $\leq 35$ ETDRS letters) and $50.6 \%(\mathrm{n}=40)$ presented criteria for low visual acuity ( $<60$ ETDRS letters). A significant increase in BCVA was observed at the 3rd month compared to baseline ( $\mathrm{p}=$ 0.001 ). At 36 months, $31.6 \%$ of the eyes had improved their BCVA (gain $\geq 5$ letters), $49.4 \%$ lost $\geq 5$ letters, and 
Fig. 3. Visual outcomes at 36 months and at the end of the follow-up.

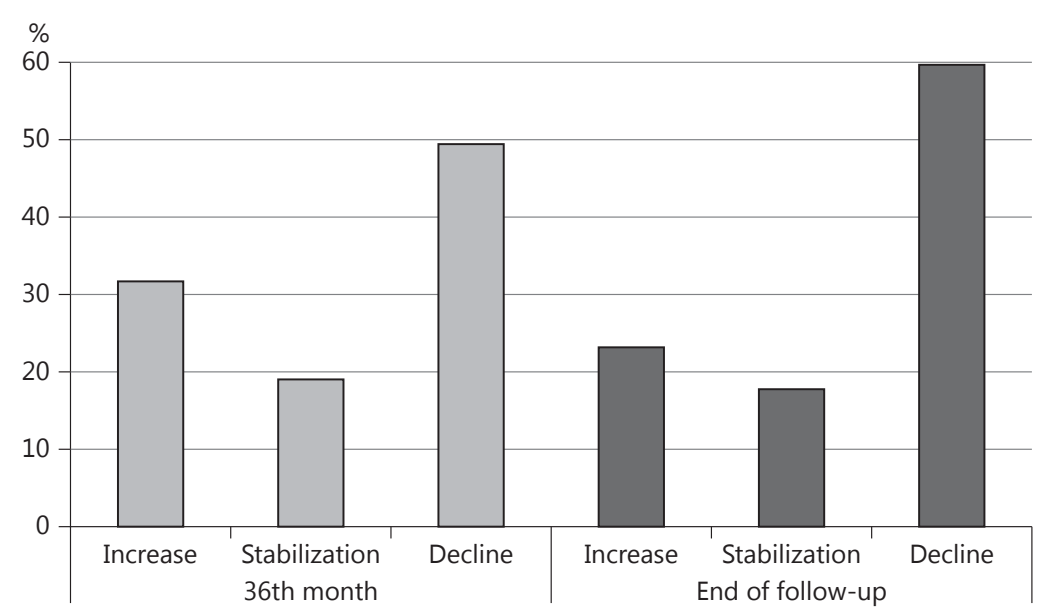

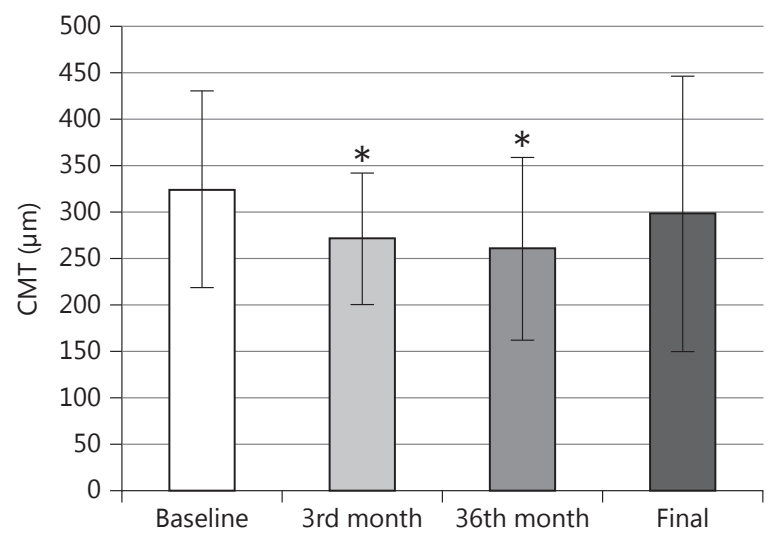

Fig. 4. CMT progression during follow-up. ${ }^{*} \mathrm{p}<0.05$.

in $19.0 \%$ there was no significant change (variation $<5$ ETDRS letters). During the whole length of follow-up, BCVA improved in $22.8 \%$, decreased in $59.5 \%$, and remained stable in $17.7 \%$. At the last visit, $53.2 \%(n=42)$ of the patients were characterized as legally blind, and $26.6 \%$ $(\mathrm{n}=21)$ were in the low vision range, whereas $20.3 \%$ ( $\mathrm{n}=$ 16) preserved a BCVA $\geq 60$ ETDRS letters. Thirty-seven eyes (46.8\%) lost $\geq 15$ letters during the whole follow-up. Mean BCVA overall variation concerning the first and last visits was $-14.0 \pm 22.7$ ( $\mathrm{p}<0.001)$. No significant differences in the visual outcome were seen between eyes treated with IV ranibizumab monotherapy and eyes treated with IV ranibizumab plus PDT $(\mathrm{p}=0.831)$. No significant correlation was found between the total number of IV ranibizumab and the BCVA variation at 36 months ( $p=0.966)$ and at the end of the follow-up ( $p=$ 0.721 compared to baseline).

\section{OCT Parameters}

Mean CMT progression during follow-up is depicted in figure 4 . There was a significant decrease in this parameter after 3 and 36 months compared to baseline ( $\mathrm{p}<$ 0.001 for both cases). Even though final CMT was still inferior to the mean baseline values, this reduction was not statistically significant $(-27.1 \pm 163.3 \mu \mathrm{m}, \mathrm{p}=0.095)$. Sixty-two eyes (78.5\%) had intra- and/or subretinal fluid at presentation. This number decreased to $58.2 \%$ at the end of the follow-up. A positive correlation was found between the presence of subretinal fluid at presentation and better final BCVA $(\mathrm{p}=0.001)$. From the 79 eyes evaluated, 47 (59.5\%) ultimately developed areas of either foveal or extrafoveal GA. No correlation was noticed between the presence of GA in the final visit and final or baseline BCVA, follow-up time or total IV ranibizumab.

\section{Treatment Parameters and Adverse Effects}

The mean number of IV ranibizumab injections per patient was $9.2 \pm 5.7$, representing a mean of $2.2 \pm 1.2$ injections/patient/year during the whole follow-up period. The highest IV ranibizumab/year ratio was observed in the first 2 years of follow-up: $3.4 \pm 1.5$ in the 1 st year and $2.2 \pm 1.4$ in the $2 \mathrm{nd}$. Thirty-four patients were treated with a combination of IV ranibizumab and PDT, while 45 un- 
derwent IV ranibizumab monotherapy. Between these two groups, no differences regarding visual outcome was found either at 36 months $(\mathrm{p}=0.816)$ or at the end of the follow-up ( $\mathrm{p}=0.699)$; sub- or intraretinal fluid at the last visit ( $\mathrm{p}=0.467$ and $\mathrm{p}=0.377$, respectively); GA development $(\mathrm{p}=0.570)$, or total number of IV ranibizumab injections ( $p=0.929)$ during the whole length of the followup. Nine patients (13.2\%) experienced vascular adverse effects after starting IV ranibizumab therapy: 7 had ischemic strokes (10.3\%). The remaining 2 patients suffered from acute myocardial infarction and pulmonary thromboembolism, respectively. All these patients had at least one systemic cardiovascular risk factor to trigger the incident.

\section{Discussion}

RAP is believed to have a worse natural history than other subtypes of exudative AMD [23]. Over the past years, the success rate of the various available treatments has been inconsistent. Nevertheless, some authors report relative stabilization of the disease with treatment, either PDT $[13,14]$ or IV ranibizumab $[18,19,21]$. PDT was combined with IV ranibizumab $[18,24]$ or even IV triamcinolone [18]. The maximal follow-up of studies on RAP treatment with IV ranibizumab with/without PDT was 3 years [18-21] and small sample sizes were small $[18,19$, $24]$. To our knowledge, this study combines the longest follow-up (59.8 \pm 16.0 months, on average) with the largest sample size $(\mathrm{n}=79)$ on RAP lesions treated with IV ranibizumab published to date.

Our clinical practice results demonstrated that PRN IV ranibizumab regimens can be effective in maintaining or improving BCVA in about half (50.6\%) of the treated eyes at 36 months, and in $40.5 \%$ after a mean follow-up of approximately 5 years. Remarkably, in about one fifth of our population reading vision was preserved at the last visit. Other studies reported better BCVA results at 2- or 3 -year follow-ups [18-20, 24]. One possible explanation could be that the use of anti-VEGF agents in clinical practice rather than in a clinical trial setting $[18,20]$ may lead to poorer than expected BCVA outcomes: the dependence on the resources and agenda of the clinical center may delay the initiation of treatment, protracting the gap between injections and subsequent follow-up. The treatment regimen - PRN - and other limitations inherent in a clinical practice setting may also have influenced our end-points, and the mean number of injections/patient/ year $(2.2 \pm 1.2)$ also suggests that our patients may have

Long-Term Management of RAP Lesions been undertreated. Furthermore, motivation may differ in a clinical trial, as the expectations of a promising new treatment modality may increase patient compliance for the scheduled visits.

No differences in visual outcome were noticed between the IV ranibizumab and IV ranibizumab plus PDT groups. Our results are in agreement with the ones reported by Rouvas et al. [18], who investigated three treatment arms: IV ranibizumab; IV ranibizumab + PDT, and IV triamcinolone + PDT. Patients were followed for 36 months and presented similar functional outcomes.

In our analysis, a significant reduction in CMT was observed at 3 months, along with a BCVA improvement, and exudation from RAP lesions seems to have regressed swiftly soon after treatment commencement. Other reports also underline this favorable initial response of RAP lesions to treatment $[19,24,25]$. The decrease in CMT between baseline and the 36-month follow-up was still statistically significant in our population. Rouvas et al. [18] also reported significant mean decreases in CMT at 3 and 36 months (last follow-up). In a recent study by Cho et al. [21], a significant decrease in mean CMT was detected after 24 months. In our cohort, CMT did not significantly differ between the last visit and baseline values. This might be explained by our longer mean follow-up time, since the treatment seems to acquire some degree of tachyphylaxis after the initial response. The high percentage of eyes with macular fluid in the final OCT (57.3\%) may be a consequence of this effect, which may reflect the final CMT values.

Those patients whose baseline OCT presented subretinal fluid experienced better visual outcomes. According to Einwallner et al. [10], the subretinal fluid plays a role in the prognosis of an exudative lesion because it also reflects its activity. These results suggest that a substantial part of the pathology of AMD is related to inflammatory processes. Ahlers et al. [26] also came to the conclusion that subretinal fluid correlated well with an improvement in BCVA, as well as with the time course of functional recovery. According to these authors, the relationship between subretinal fluid and blood-retina barrier function breakdown may explain these findings, and it may be an important parameter when defining retreatment criteria in the future. Interestingly, a recent study [27] conducted in patients enrolled in CATT (Comparison of AMD Treatments Trials) found that smaller volumes of subretinal fluid are correlated with a higher probability of GA development. Even though this was not observed in our cohort, 27 eyes (34.2\%) presented with some degree of GA at baseline, and this number rose to 47 at the last vis- 
it (59.5\%). McBain et al. [28] reported GA development in $53 \%(n=35)$ of RAP eyes, independently of the treatment chosen (IV anti-VEGF and/or PDT), a result that supports our findings. The aforementioned CATT research group paper [27] also focused on the risk factors for the development of GA and found that the RAP subtype of AMD accounted for a 1.69-fold risk of GA development. The high prevalence of GA in RAP could be explained by decreased choroidal filling in these eyes [29], thus leading to a near-ischemia status. This concept also supports the theory of an intraretinal vascular response in RAP, with budding of outward-growing retinal vessels.

This study has several limitations. Its retrospective nature prevents us from controlling variables. For example, follow-up visits should take place at each month but, due to agenda issues, this period of time was seldom respected. A higher number of patients would allow a broader analysis of GA epidemiology, which might yield conclusions with more statistical power. We do believe, however, that these limitations have otherwise a minor impact on our results, given our population's size and follow-up length.

In conclusion, our long-term follow-up on PRN IV ranibizumab for the treatment of RAP lesions in clinical practice has shown that $\sim 40 \%$ of the eyes had stable or improved BCVA at the end of our long-term follow-up, and about one fifth of the individuals preserved reading vision. Nevertheless, about $60 \%$ of the patients presented with some degree of GA at the last visit. The presence of subretinal fluid at baseline was found to positively correlate with a favorable visual outcome, thus appearing to be an important prognostic factor for functional improvement.

\section{Disclosure Statement}

R. Silva, $\mathrm{MD}, \mathrm{PhD}$, is a member of advisory boards for Allergan, Alimera, Alcon, Bayer, Novartis and Théa. J. Figueira, MD, PhD, is a member of advisory boards for Allergan, Alimera, Alcon, Bayer, Novartis and Kemin.

\section{References}

1 Gupta B, Jyothi S, Sivaprasad S: Current treatment options for retinal angiomatous proliferans (RAP). Br J Ophthalmol 2010;94:672677.

2 Hartnett ME, Weiter JJ, Garsd A, Jalkh AE: Classification of retinal pigment epithelial detachments associated with drusen. Graefes Arch Clin Exp Ophthalmol 1992;230:11-19.

3 Kuhn D, Meunier I, Soubrane G, Coscas G: Imaging of chorioretinal anastomoses in vascularized retinal pigment epithelium detachments. Arch Ophthalmol 1995;113:13921398.

4 Hartnett ME, Weiter JJ, Staurenghi G, Elsner AE: Deep retinal vascular anomalous complexes in advanced age-related macular degeneration. Ophthalmology 1996;103:20422053.

5 Slakter JS, Yannuzzi LA, Schneider U, Sorenson JA, Ciardella A, Guyer DR, et al: Retinal choroidal anastomoses and occult choroidal neovascularization in age-related macular degeneration. Ophthalmology 2000;107:742753; discussion 753-754.

6 Lafaut BA, Aisenbrey S, Vanden Broecke C, Bartz-Schmidt KU: Clinicopathological correlation of deep retinal vascular anomalous complex in age related macular degeneration. Br J Ophthalmol 2000;84:1269-1274.

7 Yannuzzi LA, Negrao S, Iida T, Carvalho C, Rodriguez-Coleman H, Slakter J, et al: Retinal angiomatous proliferation in age-related macular degeneration. Retina 2001;21:416434.
8 Lim EH, Han JI, Kim CG, Cho SW, Lee TG: Characteristic findings of optical coherence tomography in retinal angiomatous proliferation. Korean J Ophthalmol 2013;27:351-360.

9 Rouvas AA, Papakostas TD, Ntouraki A, Douvali M, Vergados I, Ladas ID: Angiographic and OCT features of retinal angiomatous proliferation. Eye (Lond) 2010;24:16331642; quiz 1643.

10 Einwallner E, Ahlers C, Golbaz I, Schutze C, Dunavolgyi R, Stock G, et al: Neovascular agerelated macular degeneration under anti-angiogenic therapy: subretinal fluid is a relevant prognostic parameter (in German). Ophthalmologe 2010;107:158-164.

11 Cohen SY, Dubois L, Tadayoni R, DelahayeMazza C, Debibie C, Quentel G: Prevalence of reticular pseudodrusen in age-related macular degeneration with newly diagnosed choroidal neovascularisation. Br J Ophthalmol 2007;91:354-359.

12 Marques JP, Lains I, Costa MA, Pires I, da Luz Cachulo M, Figueira J, Silva R: Retinal angiomatous proliferation: a quantitative analysis of the fundoscopic features of the fellow eye. Retina 2015;35:1985-1991.

13 Silva RM, Cachulo ML, Figueira J, de Abreu JR, Cunha-Vaz JG: Chorioretinal anastomosis and photodynamic therapy: a two-year follow-up study. Graefes Arch Clin Exp Ophthalmol 2007;245:1131-1139.
14 Silva RM, Faria de Abreu JR, Travassos A, Cunha-Vaz JG: Stabilization of visual acuity with photodynamic therapy in eyes with chorioretinal anastomoses. Graefes Arch Clin Exp Ophthalmol 2004;242:368-376.

15 Campa C, Harding SP, Pearce IA, Beare NA, Briggs MC, Heimann H: Incidence of neovascularization in the fellow eye of patients with unilateral retinal angiomatous proliferation. Eye (Lond) 2010;24:1585-1589.

16 Viola F, Massacesi A, Orzalesi N, Ratiglia R, Staurenghi G: Retinal angiomatous proliferation: natural history and progression of visual loss. Retina 2009;29:732-739.

17 Massacesi AL, Sacchi L, Bergamini F, Bottoni $\mathrm{F}$ : The prevalence of retinal angiomatous proliferation in age-related macular degeneration with occult choroidal neovascularization. Graefes Arch Clin Exp Ophthalmol 2008;246:89-92.

18 Rouvas AA, Chatziralli IP, Theodossiadis PG, Moschos MM, Kotsolis AI, Ladas ID: Longterm results of intravitreal ranibizumab, intravitreal ranibizumab with photodynamic therapy, and intravitreal triamcinolone with photodynamic therapy for the treatment of retinal angiomatous proliferation. Retina 2012;32:1181-1189.

19 Inoue M, Arakawa A, Yamane S, Kadonosono $\mathrm{K}$ : Long-term results of intravitreal ranibizumab for the treatment of retinal angiomatous proliferation and utility of an advanced RPE analysis performed using spectral-domain optical coherence tomography. $\mathrm{Br} \mathrm{J}$ Ophthalmol 2014;98:956-960. 
20 Gharbiya M, Parisi F, Cruciani F, BozzoniPantaleoni F, Pranno F, Abdolrahimzadeh S: Intravitreal anti-vascular endothelial growth factor for retinal angiomatous proliferation in treatment-naive eyes: long-term functional and anatomical results using a modified PrONTO-style regimen. Retina 2014;34:298305.

21 Cho HJ, Lee TG, Han SY, Kim HS, Kim JH, Han JI, et al: Long-term visual outcome and prognostic factors of intravitreal anti-vascular endothelial growth factor treatment for retinal angiomatous proliferation. Graefes Arch Clin Exp Ophthalmol 2015, Epub ahead of print.

22 Folgar FA, Jaffe GJ, Ying GS, Maguire MG, Toth CA; Comparison of Age-Related Macular Degeneration Treatments Trials Research Group: Comparison of optical coherence tomography assessments in the comparison of age-related macular degeneration treatments trials. Ophthalmology 2014;121:1956-1965.
23 Bressler NM: Retinal anastomosis to choroidal neovascularization: a bum rap for a difficult disease. Arch Ophthalmol 2005;123: 1741-1743.

24 Saito M, Iida T, Kano M: Two-year results of combined intravitreal anti-VEGF agents and photodynamic therapy for retinal angiomatous proliferation. Jpn J Ophthalmol 2013;57: 211-220.

25 Seidel G, Werner C, Weger M, Steinbrugger I, Haas A: Combination treatment of photodynamic therapy with verteporfin and intravitreal ranibizumab in patients with retinal angiomatous proliferation. Acta Ophthalmol 2013;91:e482-e485.
26 Ahlers C, Golbaz I, Stock G, Fous A, Kolar S, Pruente C, et al: Time course of morphologic effects on different retinal compartments after ranibizumab therapy in age-related macular degeneration. Ophthalmology 2008; 115:e39-e46.

27 Grunwald JE, Daniel E, Huang J, Ying GS, Maguire MG, Toth CA, et al: Risk of geographic atrophy in the comparison of age-related macular degeneration treatments trials. Ophthalmology 2014;121:150-161.

28 McBain VA, Kumari R, Townend J, Lois N: Geographic atrophy in retinal angiomatous proliferation. Retina 2011;31:1043-1052.

29 Koizumi H, Iida T, Saito M, Nagayama D, Maruko I: Choroidal circulatory disturbances associated with retinal angiomatous proliferation on indocyanine green angiography. Graefes Arch Clin Exp Ophthalmol 2008;246: 515-520. 